\title{
RETRACTED ARTICLE: High resolution seismic imaging of complex structures: a case study of the South China Sea data
}

\author{
Hao Zhang ${ }^{1}$ (D) Jun Wang ${ }^{1} \cdot$ Geping Fu ${ }^{1}$
}

Received: 29 November 2015/Accepted: 8 March 2016/Published online: 21 March 2016

(C) Springer Science+Business Media Dordrecht 2016

This article has been retracted at the request of CGG Services (Singapore) Pte Ltd. No permission was granted for the submission of this paper, which contains information, data and intellectual property belonging to the company and its client. The authors failed to comply with the journal policy because no consent had been received for submission of the work.

The online version of this article contains the full text of the retracted article as electronic supplementary material.

Electronic supplementary material The online version of this article (doi:10.1007/s11001-016-9267-2) contains supplementary material, which is available to authorized users.

\footnotetext{
Hao Zhang

zhanghaocas@qq.com; michael.zhang@cgg.com

Jun Wang

jun.wang@cgg.com

Geping Fu

geping.fu@cgg.com

1 CGG Services (Singapore) Pte Ltd, 9 Serangoon North

Avenue 5, Singapore 554531, Singapore
} 